\title{
Endophenotyping in idiopathic adult onset cervical dystonia
}

Georg Kägi ${ }^{1,2}, M D$, Diane Ruge ${ }^{1,3}$, PhD, Florian Brugger ${ }^{1,2}$, MD, Petra Katschnig ${ }^{1,4}$, MD, Rafael Sauter, Msc ${ }^{5}$, Mirta Fiorio ${ }^{6}, \mathrm{PhD}$, Michele Tinazzi ${ }^{6,7}, \mathrm{PhD}$, John Rothwell ${ }^{1}, \mathrm{MD}$, Kailash P Bhatia $^{1}, \mathrm{MD}$

1 Sobell Department of Motor Neuroscience and Movement Disorders, Institute of Neurology, Queen Square, London, United Kingdom

2 Department of Neurology, Kantonsspital St.Gallen, St.Gallen, Switzerland

3 Department of Psychology and Neurosciences, Leibniz Research Centre for

Working Environment and Human Factors at Technical University (TU), 44139 Dortmund, Germany

4 Department of Neurology, Division of Special Neurology, Medical University Graz, Graz, Austria

5 Clinical Trials Unit, Kantonsspital St. Gallen, St. Gallen, Switzerland 6 Department of Neurological and Movement Sciences, University of Verona, Italy

7 Neurology Unit, Borgo Trento Hospital, Verona, Italy

\section{e-mail addresses:}

GK: georg.kaegi@kssg.ch

DR: diane.ruge@ucl.ac.uk

FB: florian.brugger@kssg.ch

PK: petra.katschnig@medunigraz.at

RS:Rafael.Sauter@kssg.ch

MF:mirta.fiorio@univr.it

MT: michele.tinazzi@ospedaleuniverona.it

JR: j.rothwell@ucl.ac.uk

KB: k.bhatia@ucl.ac.uk

\section{Address for Correspondence:}

Georg Kägi, MD

Department of Neurology

Kantonsspital

$\mathrm{CH}-9007$ St.Gallen

Switzerland

Tel: +41 714943594

Fax: +41 714946484

e-mail: georg.kaegi@kssg.ch 


\section{HIGHLIGHTS}

- Only multimodal temporal discrimination proves to be an endophenotype of cervical dystonia (CD)

- Multimodal discrimination is abnormal in $36 \%$ of healthy $1^{\text {st }}$ degree relatives of patients with $C D$

- Unimodal discrimination, mental rotation and paired pulse TMS are unsuitable endophenotype markers 


\section{ABSTRACT}

\section{Objective}

Idiopathic adult onset cervical dystonia (IAOCD) is considered to be a partially penetrant autosomal dominant genetic condition. Dystonia may result from genetic and environmental factors. In this view, part of the physiology should be an endophenotype stemming from the genetic background. We assessed the most discriminative test to separate patients with IAOCD and healthy controls for further endophenotyping in non-affected $1^{\text {st }}$ degree relatives.

\section{Methods}

We included patients with IAOCD, their $1^{\text {st }}$ degree relatives and healthy controls. Tests performed: 1) Sensory temporal discrimination (visual, tactile, visuo-tactile), 2) Paired pulse paradigms using transcranial magnetic stimulation (TMS), 3) Mental rotation paradigms.

\section{Results}

45 patients, 18 healthy controls and 14 non-affected $1^{\text {st }}$ degree relatives were recruited. Visuotactile temporal discrimination separated best between controls and patients as well as between controls and $1^{\text {st }}$ degree relatives. $36 \%$ of the latter had an abnormal visuo-tactile temporal discrimination. No difference between patients and healthy controls was found for the other paradigms.

\section{Conclusions}

Visuo-tactile temporal discrimination separates controls from patients with IAOCD and its $1^{\text {st }}$ degree relatives. $36 \%$ of the latter had abnormal visuo-tactile thresholds supporting the role of visuo-tactile temporal discrimination as an endophenotype for IAOCD.

\section{Significance}

ur findings expand the understanding of endophenotypes in IAOCD.

Key words: dystonia, temporal discrimination, transcranial magnetic stimulation, mental rotation, endophenotype 


\section{INTRODUCTION}

Dystonia is a movement disorder characterized by sustained or intermittent muscle contractions causing abnormal, often repetitive, movements, postures, or both. (Albanese et al., 2013) Idiopathic adult onset cervical dystonia (IAOCD) is considered to be an autosomal dominant genetic condition due to partially penetrant gene/s.(Stojanovic et al., 1995; Waddy et al., 1991) Hence most of the cases appear to be sporadic with a positive family history in $15-25 \%$.(Waddy et al., 1991) Several genes have been mapped for families, which include members with cervical dystonia (Almasy et al., 1997; Charlesworth et al., 2012; Fuchs et al., 2009; Fuchs et al., 2013; Leube et al., 1996; Valente et al., 2001; Xiao et al., 2012) but in several other families with cervical dystonia linkage to these known genetic loci has been excluded. Like in many disorders, dystonia is considered to be caused by a combination of genetic factors and environmental modifiers. In this view, at least part of the physiology should be a trait stemming from the genetic background.(Hallett 2011)

The identification of the genetic causation has been difficult because informative families are rare due to low penetrance of the phenotype and absence of surrogate markers. One approach to find the gene(s) is to find a sensitive endophenotype, "a marker of subclinical gene carriage" in non-affected relatives. A useful model to study endophenotype markers is inherited generalized dystonia (DYT1) because penetrance is low. Compared to controls, both, manifesting and non-manifesting DYT1 gene mutation carriers share common features like deficient inhibition on a cortical level,(Edwards et al., 2003) impaired sensory temporal processing,(Fiorio et al., 2007a) and impaired body movement representation in the mental rotation task.(Fiorio et al., 2008)

Several of these characteristics can also be found in patients with cervical dystonia:

i) sensory discrimination: studies have shown that spatial (Molloy et al., 2003; O'Dwyer et al., 2005; Walsh et al., 2007) and temporal sensory discrimination (Bradley et al., 2009; Scontrini et al., 2009; Tinazzi et al., 2004; Kimmich et al., 2014) is impaired in patients with cervical dystonia with the latter being more discriminative.(Bradley et al., 2009; Bradley et al., 2010; Walsh et al., 
2007) Impaired sensory discrimination has been found in $1^{\text {st }}$ degree relatives of patients with cervical dystonia (Bradley et al., 2009; Walsh et al., 2007; Kimmich et al., 2014)

ii) impaired inhibition: Impaired intracortical inhibition using transcranial magnetic stimulation is present in both hemispheres (ipsi-and contralateral to the dystonic hand) suggesting that this abnormality is likely to be a substrate or trait for dystonia.(Ridding et al., 1995a) In cervical dystonia, there are conflicting reports with reduced short interval intracortical inhibition only when measured from the sternocleidomastoid muscle or only when magnetic stimulation was performed on the contralateral side of head deviation.(Hanajima et al., 1998; Kanovsky et al., 2003)

iii) The mental rotation paradigm: Similar to real movements, mentally simulated movements require an intact specific cortico-subcortical motor network(lonta et al., 2007) as well as intact sensory systems.(Cohen et al., 1996; Vingerhoets et al., 2002) Patients with idiopathic dystonia are slower in mentally rotating corporal objects.(Fiorio et al., 2006; Fiorio et al., 2007b; Fiorio et al., 2008) In patients with focal hand dystonia, slower mental rotation was observed on the affected and unaffected side.(Fiorio et al., 2006)

The aim of this study was to determine the most discriminative test between IAOCD and healthy controls with the idea that an endophenotype marker should be present in subjects presenting with the phenotype and absent in controls. According to the literature we selected temporal sensory discrimination, short intracortical inhibition and mental rotation paradigms. In a second step we applied the test(s) discriminating patients from controls to non-affected $1^{\text {st }}$ degree relatives to decide whether it is part of the phenotype or an endophenotype.

\section{PATIENTS AND METHODS}

We recruited patients with IAOCD who were attending the Movement disorders or botulinum toxin clinics at the National Hospital for Neurology and Neurosurgery, London, their healthy first degree relatives as well as healthy controls without a family history of dystonia. After giving written informed consent, eligible subjects were clinically examined by an experienced Movement disorders specialist (GK). Participants with clinical evidence for polyneuropathy or 
carpal tunnel syndrome were excluded. Dystonia severity was quantified with the Toronto Western Spasmodic Torticollis Rating Scale (TWSTRS). The study was approved by the local research ethics committee. (Katschnig et al., 2010)

\subsection{Temporal discrimination thresholds}

Sensory temporal discrimination thresholds (TDT) were assessed by pairs of visual, tactile and visuo-tactile (crossmodal) stimuli according to the published protocol.(Fiorio et al., 2003; Kagi et al., 2013) In short, pairs of stimuli were delivered with progressive increase of the interstimulus interval (ISI). The order of the stimulation paradigms was randomized. Visual stimuli were delivered through light-emitting-diodes (LED) positioned on a black board. Two LEDs in each visual field at $7^{\circ}$ from central fixation point. Tactile stimulation consisted of electrical stimuli (constant current) through surface electrodes positioned on the volar side of the index and middle finger of the right and left hand. The anodes were located $1.5 \mathrm{~cm}$ distally to the cathodes. Stimulation intensity for tactile stimulation was determined for each subject starting at $2 \mathrm{~mA}$ and an increment of $1 \mathrm{~mA}$ unless 10 out of 10 stimuli were perceived. Each run started with an ISI of Oms with progressive increase by steps of $10 \mathrm{~ms}$. TDT was considered the first (i.e. the shortest) out of three consecutive ISI at which the two stimuli were recognized as asynchronous. Each run was repeated 4 times with the mean taken as the TDT. Additionally, subjects were asked to judge, which stimulus preceded (or followed) the other. The first (i.e. shortest) out of three consecutive ISI at which subjects also reported correctly the temporal order in a pair of stimuli was termed temporal order judgment (TOJ). Again the mean of 4 runs was taken for further analysis.

\subsection{Transcranial magnetic stimulation}

Subjects were seated with the hands relaxed (audiovisually controlled) on a pillow. Surface electrodes were placed on the FDI muscle of the dominant hand. Stimulation was performed using 2 Magstim 200 stimulators and a figure-of eight-shaped coil, external wing diameter $9 \mathrm{~cm}$ (Magstim, Dyfed, UK). The coil handle was positioned to evoke an anteriorly directed current in 
the brain. Optimal coil position was defined as the position with the lowest threshold to evoke a response in the FDI muscle of the contralateral hand. Resting motor threshold was then defined as the intensity to evoke MEPs ( $>50 \mathrm{uV}$ amplitude) in more than 5 out of 10 runs. Active motor threshold was defined likewise but with a minimal background muscle activity $(10-15 \%$ of maximum). Test MEP intensity was set in order to evoke a MEP of $\sim 1 \mathrm{mV}$. Short intracortical inhibition-/facilitation paradigms followed the protocol described by Kujirai et al.(Kujirai et al., 1993) We used interstimulus intervals of 2 and 3ms for short intracortical inhibition, of 6 and $8 \mathrm{~ms}$ for intermediate and of $10,12,15 \mathrm{~ms}$ for intracortical fascilitation. The intensity of the conditioning stimulus was set at $80 \%$ of active motor threshold. For short intracortical inhibitionrecruitment curve a fixed interstimulus interval of $2 \mathrm{~ms}$ with increasing intensity of the conditioning stimulus $(70 \%, 80 \%, 90 \%$ of test pulse intensity) was used. The percentage $\left((\mathrm{cMEP} / \mathrm{tMEP})^{*} 100\right)$ was used for further analysis. Experimental conditions were presented 12 times each.

\subsection{Mental rotation}

The mental rotation paradigm was adapted from previous studies in idiopathic dystonia.(Fiorio et al., 2006; Fiorio et al., 2007b; Fiorio et al., 2008) In brief, subjects were presented with realistic photos of left or right hands, feet and a head of a woman with a black patch covering either the left or right eye. All three stimuli were presented in six angular orientations $(0,60$, $120,180,240$, and 300 ) and subjects had to verbally report the laterality of the presented stimuli. Reaction time (RT) was recorded by a microphone and response accuracy was entered into the computer by the researcher. Only RTs with correct laterality judgment were considered for further analysis. The ratio of correct responses to the total number of stimuli was used as error rate.

\subsection{Statistics}

Data were analyzed using PASW Statistics 20 for Windows and R statistical software (version 2.15.3 (2013-03-01). For the groups, absolute (n) and relative (\%) frequencies are presented for 
nominal and mean with standard deviation for continuous patient characteristics. $X^{2}$ test was applied for gender and handedness and one way ANOVA for age, age of onset, disease duration, handedness, TWSTRS score and subscores I-III.

In a next step, we used a receiver operating characteristic $(\mathrm{ROC})$ curve for the temporal sensory discrimination paradigms to identify the most accurate test to discriminate between patients and healthy controls. The only discriminative test between patients and controls (visuotactile paradigm) was further tested by ROC and linear regression analysis between controls and non-affected first degree relatives. To account for possible confounders we included "age" and "education" as co-variables in a linear regression model with "visuo-tactile TDT" as dependent variable and "patient-group" as "factor".

Because ROC interpretation is difficult with low sample sizes, we added the Mann-Whithney-U test to compare TMS measures ( $\mathrm{SICI}, \mathrm{IM}, \mathrm{ICF}, \mathrm{SICl}_{70}, \mathrm{SICl}_{80}, \mathrm{SICl}_{90}$ ) between patients and controls.

ANOVA for repeated measures was applied for the mental rotation paradigm. Reaction times were analyzed with one between-subject factor 'group' (patients and controls) and three withinsubject factors 'stimulus orientation' (0,60, 120, 180, 240, 300), 'stimulus side' (right, left) and 'error rate'.

In this exploratory study the error rate was not controlled at a pre-specified significance level and no correction for multiple testing was considered. However, if appropriate, we provide pvalues derived from the corresponding statistical tests and assume that the available data provide evidence against the null hypothesis if the $p$-value is below $5 \%$.

\section{RESULTS}

45 patients with cervical dystonia, 18 healthy controls and 14 non-affected $1^{\text {st }}$ degree relatives were recruited. There was a significant difference in age between the groups $\left(F_{2,7}=. ; p<0.0001\right)$ due to and non-affected $1^{\text {st }}$ degree relatives being younger compared to IAOCD(post-hoc t-test uncorrected $(p=0.032$ and $p<0.0001) \quad(p=0.0)$. No sA significant differences were found for education $\left(F_{2,}=.\right)$, gender $(p=0$.) and handedness $(p=0$.). (Table 1) 


\subsection{Temporal sensory discrimination}

The visuo-tactile paradigm was the paradigm discriminating between patients and controls TDT (AUC ) (Figure 1); TOJ (AUC $\left.0.81\left[\mathrm{Cl}_{95} 0 ., 0.\right]\right)$. This was not the case for visual and tactile

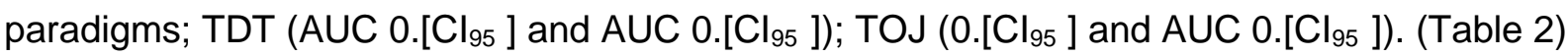
Because TOJ data didn't perform better than TDT data we used the latter for further analysis. Calculating $\mathrm{J}$ statistics from the visuo-tactile paradigm in healthy controls gives a maximum Youden-Index of 0. at a threshold of with a specificity of \% $\left(\mathrm{Cl}_{95} 0 ., 0.9\right)$ and sensitivity of \% $\left(\mathrm{Cl}_{95}\right.$ 0., 0.). At a specificity level of .\% sensitivity is $56 \%$ (cut off:.). This threshold was reached in five out of $14(36 \%)$ non-affected relatives. ROC analysis of the visuo tactile TDT between controls and non-affected relatives was borderline with an AUC of $0.7\left[\mathrm{Cl}_{95} 0.48,0.85\right]$.

The linear regression model with "visuo-tactile TDT" as dependent and "age" and "education" as co-variables revealed a significant difference not only between patients and controls $\left(\mathrm{Cl}_{95}[\right.$,$] ,$ $\mathrm{p}<0.0001)$ but also between controls and non-affected relatives $\left(\mathrm{Cl}_{95}[0 .],, \mathrm{p}=0.\right)$. Education (years) showed a negative correlation with visuo-tactile TDT (Spearman's $r:-0.234 ; p=0.033$ ) in patients and controls.

\subsection{Transcranial magnetic stimulation}

Due to recruitment issues only 21 patients and 8 controls could be tested. All patients and 7/8 controls had all tests (sensory testing, TMS, mental rotation). Descriptives of these groups didn't show significant differences except that the patients had 3.7 years less education (14 y vs 17.7 years; $p=0.024$ ). Neither ROC curves nor Mann-Whithney $U$ test for $\mathrm{SICI}, \mathrm{IM}, \mathrm{ICF}, \mathrm{SICl}_{70}$, $\mathrm{SICl}_{80}, \mathrm{SICl}_{90}$ showed differences between patients from controls. (Table 3)

\subsection{Mental rotation}

37/45 patients and 15 controls were studied. . The ANOVA for reaction times was not significant for the factor 'group' $\left(F_{1,}=, p=0\right.$.) although significant effects were seen for the factor 'degree' $\left(F_{5,}=, p<0.00001\right)$, 'region' $\left(F_{2,}=, p<0.00001\right)$ and 'error rate' $\left(F_{3,6461}=6.95, p=0.0084\right)$. Post 
hoc tests for 'degree' showed significant differences for $120^{\circ}(p<0.00001), 180^{\circ}(p<0.00001)$ and $240^{\circ}(p=4)$ due to longer reaction times for $120^{\circ}(+\mathrm{ms}), 180^{\circ}(+\mathrm{ms})$ and $240^{\circ}(+\mathrm{ms})$ compared to the reference angle $\left(0^{\circ}\right)$. The differences for the factor 'region' were due to a shorter reaction time for the head $(-\mathrm{ms} ; \mathrm{p}<0.00001))$ compared to the feet. The differences for the factor 'error rate' were due to longer reaction times $(+126.6 \mathrm{~ms})$ in subjects with errors $(p=0.0002)$ compared to those without.

\section{DISCUSSION}

This study confirms previous reports of impaired temporal discrimination in patients with cervical dystonia.(Bradley et al., 2009; Scontrini et al., 2009; Tinazzi et al., 2004; Kimmich et al., 2014; Kagi et al., 2013) 5 out of $14(36 \%)$ non-affected $1^{\text {st }}$ degree relatives had abnormal visuotactile temporal discrimination thresholds. Considering that only $50 \%$ of $1^{\text {st }}$ degree relatives are expected to be gene mutation carriers, the finding supports previous reports that subtle sensory abnormalities represent a trait or endophenotype of gene mutation carriage. In addition, Kimmich et al showed that in first degree relatives temporal discrimination exhibits a comparable prevalence compared to the phenotype reaching full penetrance after the age of 48 years.(Kimmich et al., 2014)

The fact that the latter research group found the largest differences in the visual and tactile compared to mixed (visuo-tactile) paradigm is intriguing and hard to explain.(Bradley et al., 2009; Kimmich et al., 2014) (Tinazzi et al., 2002)patients with IAOCD multimodal (visuo-tactile) temporal discrimination might be more affected compared to the unimodal temporal discrimination; i) the parietal lobe as an important center of multimodal sensory integration is part of the dystonia network(Lacruz et al., 1991; Leon and Shadlen, 2003; Neychev et al., 2011; Pastor et al., 2004), ii) this notion receives support by the finding that patients with a good effect to a sensory trick perform significantly better in the visuo-tactile sensory discrimination and that the parietal lobe is activated while performing an effective sensory trick (Kagi et al., 2013; Naumann et al., 2000), iii) the superior colliculi, where multisensory (visual, tactile, auditory) 
inputs converge are involved in head-turn generation with its cephalomotor premotor neurons (Hutchinson et al., 2014).

Several previous studies have shown that inhibition is defective within and distant to the affected body region in idiopathic adult onset focal dystonia.(Berardelli et al., 1998; Chen et al., 1997; Hallett2011; Ridding et al., 1995a) In line with most of the literature on short interval intracortical inhibition in cervical dystonia, our study failed to show any difference between patients and controls and therefore doesn't appear to be suitable as endophenotype marker. Due to the fact that the number for controls was rather low $(n=8)$ this notion has to be interpreted with caution. There are several reasons for the variable results reported for short intracortical inhibition in cervical dystonia. For example, intracortical inhibition is reduced by minimal voluntary muscle activation even in muscles remote from the target. Since some patients will have neck movement during assessment this will significantly increase the variability of the patient data.(Ridding et al., 1995b) In addition it should be noted that even in healthy participants, paired-pulse TMS paradigms have an large intersubject variability of up to 80\%.(Wassermann 2002)

The mental rotation paradigm didn't separate patients from controls. The ANOVA model failed to confirm the differences found between patients and healthy controls in previous studies using the same paradigm, methodological setting and with the same statistics.(Fiorio et al., 2007b; Katschnig et al., 2010) Compared to these two studies the patients were within the same range but the controls performed worse. Since only controls but not patients performed worse, it makes methodological reasons unlikely to account for the differences. For the purpose of our study, mental rotation does not represent a valuable endophenotype marker.

What is the value of temporal sensory discrimination testing in $1^{\text {st }}$ degree relatives for genetic testing?

Although subtle sensory abnormalities seem to represent a trait of dystonia, its value for genetic studies is limited. Imagine we are investigating one family with dystonia in whom there are 5 affected members. For genetic studies we would need another 5 non-affected first degree 
relatives who are correctly selected (true positives) by a given test. In brief, to get 5 true positives we would need to test 20 first degree relatives (because we expect 10 to be non-gene mutation carriers due to the autosomal dominant inheritance). It is important to set the specificity near $100 \%$ because in genetic linkage studies the required "logarithm of odds" (LOD) score of $>+3$ (evidence for linkage) is achieved in 10 true positive samples. If 1 sample is a false positive the LOD score decreases to around +1.5 . In our 14 non-affected first degree relatives (we assume that half (7) are mutation carriers) we would have recruited 5 subjects with the specificity set at $95.7 \%$.

In conclusion, only crossmodal (visuo-tactile) temporal sensory discrimination separated healthy controls from patients as well as from their non-affected $1^{\text {st }}$ degree relatives. Almost $40 \%$ of the latter had abnormal visuo-tactile temporal discrimination thresholds supporting its role as an endophenotype marker for IAOCD. Unimodal (visual, tactile) temporal discrimination, intracortical inhibition/facilitation or mental rotation did not separate patients from healthy controls making them unsuitable for endophenotype markers.

\section{ACKKNOWLEGEMENT}

For this work GK was supported by the Swiss Parkinson's Disease Association and the Halley Stewart Trust. The funding sources haven't been involved in data collection, analysis and interpretation of data or writing the manuscript.

Conflict of interest: None of the authors have potential conflicts of interest to be disclosed.

\section{REFERENCES}


Albanese A, Bhatia K, Bressman SB, Delong MR, Fahn S, Fung VS, et al. Phenomenology and classification of dystonia: A consensus update. Mov Disord 2013; 28: 863-873.

Almasy L, Bressman SB, Raymond D, Kramer PL, Greene PE, Heiman GA, et al. Idiopathic torsion dystonia linked to chromosome 8 in two Mennonite families. Ann Neurol 1997; 42: 670673.

Berardelli A, Rothwell JC, Hallett M, Thompson PD, Manfredi M, Marsden CD. The pathophysiology of primary dystonia. Brain 1998; 121 ( Pt 7): 1195-1212.

Bradley D, Whelan R, Walsh R, O'Dwyer J, Reilly R, Hutchinson S, et al. Comparing endophenotypes in adult-onset primary torsion dystonia. Mov Disord 2010; 25: 84-90.

Bradley D, Whelan R, Walsh R, Reilly RB, Hutchinson S, Molloy F, et al. Temporal discrimination threshold: VBM evidence for an endophenotype in adult onset primary torsion dystonia. Brain 2009; 132: 2327-2335.

Charlesworth G, Plagnol V, Holmstrom KM, Bras J, Sheerin UM, Preza E, et al. Mutations in ANO3 Cause Dominant Craniocervical Dystonia: Ion Channel Implicated in Pathogenesis. Am J Hum Genet 2012; 91: 1041-1050.

Chen R, Wassermann EM, Canos M, Hallett M. Impaired inhibition in writer's cramp during voluntary muscle activation. Neurology 1997; 49: 1054-1059.

Cohen MS, Kosslyn SM, Breiter HC, DiGirolamo GJ, Thompson WL, Anderson AK, et al. Changes in cortical activity during mental rotation. A mapping study using functional MRI. Brain 1996; 119 ( Pt 1): 89-100.

Edwards MJ, Huang YZ, Wood NW, Rothwell JC, Bhatia KP. Different patterns of electrophysiological deficits in manifesting and non-manifesting carriers of the DYT1 gene mutation. Brain 2003; 126: 2074-2080.

Fiorio M, Gambarin M, Defazio G, Valente EM, Stanzani C, Moretto G, et al. Impaired body movement representation in DYT1 mutation carriers. Clin Neurophysiol 2008; 119: 1864-1869.

Fiorio M, Gambarin M, Valente EM, Liberini P, Loi M, Cossu G, et al. Defective temporal processing of sensory stimuli in DYT1 mutation carriers: a new endophenotype of dystonia? Brain 2007a; 130: 134-142.

Fiorio M, Tinazzi M, Aglioti SM. Selective impairment of hand mental rotation in patients with focal hand dystonia. Brain 2006; 129: 47-54.

Fiorio M, Tinazzi M, Bertolasi L, Aglioti SM. Temporal processing of visuotactile and tactile stimuli in writer's cramp. Ann Neurol 2003; 53: 630-635.

Fiorio M, Tinazzi M, lonta S, Fiaschi A, Moretto G, Edwards MJ, et al. Mental rotation of body parts and non-corporeal objects in patients with idiopathic cervical dystonia. Neuropsychologia 2007b; 45: 2346-2354.

Fuchs T, Gavarini S, Saunders-Pullman R, Raymond D, Ehrlich ME, Bressman SB, et al. Mutations in the THAP1 gene are responsible for DYT6 primary torsion dystonia. Nat Genet 2009; 41: 286-288.

Fuchs T, Saunders-Pullman R, Masuho I, Luciano MS, Raymond D, Factor S, et al. Mutations in GNAL cause primary torsion dystonia. Nat Genet 2013; 45: 88-92. 
Hallett M. Neurophysiology of dystonia: The role of inhibition. Neurobiol Dis 2011; 42: 177-184.

Hanajima R, Ugawa Y, Terao Y, Sakai K, Furubayashi T, Machii K, et al. Cortico-cortical inhibition of the motor cortical area projecting to sternocleidomastoid muscle in normals and patients with spasmodic torticollis or essential tremor. Electroencephalogr Clin Neurophysiol 1998; 109: 391-396.

Hutchinson M, Isa T, Molloy A, Kimmich O, Williams L, Molloy F, et al. Cervical dystonia: a disorder of the midbrain network for covert attentional orienting. Front Neurol 2014; 5: 54.

Ionta S, Fourkas AD, Fiorio M, Aglioti SM. The influence of hands posture on mental rotation of hands and feet. Exp Brain Res 2007; 183: 1-7.

Kagi G, Katschnig P, Fiorio M, Tinazzi M, Ruge D, Rothwell J, et al. Sensory tricks in primary cervical dystonia depend on visuotactile temporal discrimination. Mov Disord 2013; 28: 356-361.

Kanovsky P, Bares M, Streitova H, Klajblova H, Daniel P, Rektor I. Abnormalities of cortical excitability and cortical inhibition in cervical dystonia Evidence from somatosensory evoked potentials and paired transcranial magnetic stimulation recordings. J Neurol 2003; 250: 42-50.

Katschnig P, Edwards MJ, Schwingenschuh P, Aguirregomozcorta M, Kagi G, Rothwell JC, et al. Mental rotation of body parts and sensory temporal discrimination in fixed dystonia. Mov Disord 2010; 25: 1061-1067.

Kimmich O, Molloy A, Whelan R, Williams L, Bradley D, Balsters J, et al. Temporal discrimination, a cervical dystonia endophenotype: penetrance and functional correlates. Mov Disord 2014; 29: 804-811.

Kujirai T, Caramia MD, Rothwell JC, Day BL, Thompson PD, Ferbert A, et al. Corticocortical inhibition in human motor cortex. J Physiol 1993; 471: 501-519.

Lacruz F, Artieda J, Pastor MA, Obeso JA. The anatomical basis of somaesthetic temporal discrimination in humans. J Neurol Neurosurg Psychiatry 1991; 54: 1077-1081.

Leon MI, Shadlen MN. Representation of time by neurons in the posterior parietal cortex of the macaque. Neuron 2003; 38: 317-327.

Leube B, Rudnicki D, Ratzlaff T, Kessler KR, Benecke R, Auburger G. Idiopathic torsion dystonia: assignment of a gene to chromosome $18 p$ in a German family with adult onset, autosomal dominant inheritance and purely focal distribution. Hum Mol Genet 1996; 5: 16731677.

Molloy FM, Carr TD, Zeuner KE, Dambrosia JM, Hallett M. Abnormalities of spatial discrimination in focal and generalized dystonia. Brain 2003; 126: 2175-2182.

Naumann M, Magyar-Lehmann S, Reiners K, Erbguth F, Leenders KL. Sensory tricks in cervical dystonia: perceptual dysbalance of parietal cortex modulates frontal motor programming. Ann Neurol 2000; 47: 322-328.

Neychev VK, Gross RE, Lehericy S, Hess EJ, Jinnah HA. The functional neuroanatomy of dystonia. Neurobiol Dis 2011; 42: 185-201.

O'Dwyer JP, O'Riordan S, Saunders-Pullman R, Bressman SB, Molloy F, Lynch T, et al. Sensory abnormalities in unaffected relatives in familial adult-onset dystonia. Neurology 2005; 65: 938-940. 
Pastor MA, Day BL, Macaluso E, Friston KJ, Frackowiak RS. The functional neuroanatomy of temporal discrimination. J Neurosci 2004; 24: 2585-2591.

Ridding MC, Sheean G, Rothwell JC, Inzelberg R, Kujirai T. Changes in the balance between motor cortical excitation and inhibition in focal, task specific dystonia. J Neurol Neurosurg Psychiatry 1995a; 59: 493-498.

Ridding MC, Taylor JL, Rothwell JC. The effect of voluntary contraction on cortico-cortical inhibition in human motor cortex. J Physiol 1995b; 487 ( Pt 2): 541-548.

Scontrini A, Conte A, Defazio G, Fiorio M, Fabbrini G, Suppa A, et al. Somatosensory temporal discrimination in patients with primary focal dystonia. J Neurol Neurosurg Psychiatry 2009; 80: $1315-1319$.

Stojanovic M, Cvetkovic D, Kostic VS. A genetic study of idiopathic focal dystonias. J Neurol 1995; 242: 508-511.

Tinazzi M, Fiaschi A, Frasson E, Fiorio M, Cortese F, Aglioti SM. Deficits of temporal discrimination in dystonia are independent from the spatial distance between the loci of tactile stimulation. Mov Disord 2002; 17: 333-338.

Tinazzi M, Fiorio M, Bertolasi L, Aglioti SM. Timing of tactile and visuo-tactile events is impaired in patients with cervical dystonia. J Neurol 2004; 251: 85-90.

Valente EM, Bentivoglio AR, Cassetta E, Dixon PH, Davis MB, Ferraris A, et al. DYT13, a novel primary torsion dystonia locus, maps to chromosome 1p36.13--36.32 in an Italian family with cranial-cervical or upper limb onset. Ann Neurol 2001; 49: 362-366.

Vingerhoets G, de Lange FP, Vandemaele P, Deblaere K, Achten E. Motor imagery in mental rotation: an fMRI study. Neuroimage 2002; 17: 1623-1633.

Waddy HM, Fletcher NA, Harding AE, Marsden CD. A genetic study of idiopathic focal dystonias. Ann Neurol 1991; 29: 320-324.

Walsh R, O'Dwyer JP, Sheikh IH, O'Riordan S, Lynch T, Hutchinson M. Sporadic adult onset dystonia: sensory abnormalities as an endophenotype in unaffected relatives. J Neurol Neurosurg Psychiatry 2007; 78: 980-983.

Wassermann EM. Variation in the response to transcranial magnetic brain stimulation in the general population. Clin Neurophysiol 2002; 113: 1165-1171.

Xiao J, Uitti RJ, Zhao Y, Vemula SR, Perlmutter JS, Wszolek ZK, et al. Mutations in CIZ1 cause adult onset primary cervical dystonia. Ann Neurol 2012; 71: 458-469. 
TABLES AND FIGURES

TABLE 1: Patient characteristics

\begin{tabular}{|l|l|l|l|}
\hline & IAOCD & $1^{\text {st o } \text { relatives }}$ & controls \\
\hline number & 45 & 14 & 23 \\
\hline gender (\% females) & 64.4 & 75 & 56.5 \\
\hline $\begin{array}{l}\text { handedness }(\% \\
\text { right) }\end{array}$ & 86.7 & 100 & 95.7 \\
\hline age (y) & $56.3 \pm 10.9$ & $38.7 \pm 14.7$ & $48.9 \pm 16.8$ \\
\hline age of onset $(\mathrm{y})$ & $41.5 \pm 14.1$ & --- & --- \\
\hline education $(\mathrm{y})$ & 14.2 & 14.9 & 16.7 \\
\hline disease duration $(\mathrm{y})$ & $14.5 \pm 10.3$ & --- & --- \\
\hline TWSTRS & & & \\
\hline total & $23.9 \pm 10.4$ & --- & --- \\
\hline subscore I & $11.8 \pm 3.9$ & --- & --- \\
\hline subscore II & $7.7 \pm 4.2$ & --- & --- \\
\hline subscore III & $5.2 \pm 4.7$ & --- & --- \\
\hline
\end{tabular}

IAOCD: idiopathic adult onset cervical dystonia. TWSTRS: Toronto Western Spasmodic

Torticollis Rating Scale. Data are presented as mean with standard deviation if not stated otherwise 
TABLE 2: Temporal discrimination thresholds

\begin{tabular}{|c|c|c|c|c|}
\hline & IAOCD & $1^{\text {st }}$ relatives & controls & $\begin{array}{c}\text { ROC, AUC [CI(95\%)] } \\
\text { IAOCD vs controls }\end{array}$ \\
\hline $\mathrm{n}$ & 45 & 14 & 23 & \\
\hline visual tdt & $54.2 \pm 18.2$ & $40.6 \pm 10.8$ & $42.4 \pm 16.2$ & $0.68[0.54 ; 0.82]$ \\
\hline visual toj & $56.1 \pm 18.7$ & $43.5 \pm 12.6$ & $44.8 \pm 17.0$ & $0.68[0.54 ; 0.82]$ \\
\hline tactile tdt & $89.6 \pm 42.9$ & $70.1 \pm 27.5$ & $63.7 \pm 27.9$ & $0.69[0.56 ; 0.83]$ \\
\hline tactile toj & $100.0 \pm 51.2$ & $79 \pm 33.5$ & $71.7 \pm 32.2$ & $0.67[0.53 ; 0.81]$ \\
\hline cm tdt & $140.6 \pm 49.5$ & $115.8 \pm 35.8$ & $89.8 \pm 25.3$ & $0.85[0.76 ; 0.94]$ \\
\hline cm toj & $146.1 \pm 49.6$ & $127 \pm 31.1$ & $99.9 \pm 30.9$ & $0.81[0.70 ; 0.92]$ \\
\hline
\end{tabular}

IAOCD: idiopathic adult onset cervical dystonia.cm: crossmodal (visuo-tactile), tdt: temporal discrimination threshold in milliseconds, toj: temporal order judgment in milliseconds, Cl: confidence interval, ROC: Receiver operating characteristic, AUC: area under the curve. The values are reported as mean with standard deviation. 
TABLE 3: Short intracortical inhibition (SICI), intracortical fascilitation (ICF)

\begin{tabular}{|l|l|l|l|}
\hline & IAOCD & controls & $p$-value* \\
\hline $\mathrm{n}$ & 21 & 8 & \\
\hline $\mathrm{SICI} 70 \%$ & $81.1 \pm 33.1$ & $103.1 \pm 67.6$ & 0.340 \\
\hline $\mathrm{SICI} 80 \%$ & $63.6 \pm 32.9$ & $72.2 \pm 28.5$ & 0.374 \\
\hline $\mathrm{SICI} 90 \%$ & $63.6 \pm 55.1$ & $52.6 \pm 26.5$ & 0.783 \\
\hline $\mathrm{SICI}$ & $49.6 \pm 26.2$ & $53.4 \pm 37.1$ & 0.850 \\
\hline Intermediate & $102.9 \pm 44.4$ & $101.4 \pm 30.2$ & 1 \\
\hline ICF & $122.7 \pm 56.7$ & $126.1 \pm 41.4$ & 0.613 \\
\hline
\end{tabular}

IAOCD: idiopathic adult onset cervical dystonia. SICl: short intra-cortical inhibition, ICF:

intracortical facilitation. The values are presented as mean with standard deviation. *Mann-

Whitney-U-Test 
Supplemental Table 1: Mean reaction times (ms) in patients and controls for the three body-stimuli (hand, feet, head), and their laterality (left and right) and orientations $(0 \circ, 60 \circ, 120 \circ, 180 \circ, 240 \circ, 300 \circ)$

\begin{tabular}{|c|c|c|c|c|c|c|c|c|c|c|c|c|}
\hline & \multicolumn{6}{|l|}{ Right } & \multicolumn{6}{|l|}{ Left } \\
\hline & $0^{\circ}$ & $60^{\circ}$ & $120^{\circ}$ & $180^{\circ}$ & $240^{\circ}$ & $300^{\circ}$ & $0^{\circ}$ & $60^{\circ}$ & $120^{\circ}$ & $180^{\circ}$ & $240^{\circ}$ & $300^{\circ}$ \\
\hline \multicolumn{13}{|l|}{ Hand } \\
\hline IAOCD & 2118 & 2266 & 2586 & 2773 & 2071 & 1907 & 2082 & 1960 & 2036 & 2670 & 2276 & 2091 \\
\hline controls & 1768 & 1915 & 2126 & 2332 & 1843 & 1848 & 1881 & 1644 & 1922 & 2260 & 2088 & 1846 \\
\hline IAOCD & 1847 & 1741 & 1938 & 1957 & 1813 & 1814 & 1770 & 1789 & 1827 & 2225 & 1726 & 1768 \\
\hline controls & 1659 & 1553 & 1741 & 1965 & 1793 & 1627 & 1481 & 1451 & 1590 & 1791 & 1564 & 1446 \\
\hline \multicolumn{13}{|l|}{ Feet } \\
\hline IAOCD & 2039 & 2042 & 2391 & 2565 & 2337 & 2109 & 2003 & 2153 & 2390 & 2550 & 2114 & 2042 \\
\hline
\end{tabular}

IAOCD: idiopathic adult onset cervical dystonia. ms: milliseconds. 


\section{Figure 1:}

Title: ROC curves for different temporal discrimination modalities in patients with IAOCD vs. healthy controls

Legend: tactile_tdt: tactile temporal discrimination thresholds, visual_tdt: visual temporal discrimination thresholds, crossm_tdt: crossmodal (visuo-tactile) temporal discrimination thresholds 